\title{
Development and internal validation of nomograms for the prediction of postoperative survival of patients with grade 4 renal cell carcinoma (RCC)
}

\author{
Jun Zhu ${ }^{1,2,3 \#}$, Zhifu Liu ${ }^{1,2,3 \#}$, Zhongyuan Zhang ${ }^{1,2,3 \#}$, Yu Fan ${ }^{1,2,3}$, Yuke Chen ${ }^{1,2,3}$, Zhisong He , $^{1,2,3}$ \\ Liqun Zhou ${ }^{1,2,3}$, Jie Jin ${ }^{1,2,3}$, Cheng Shen ${ }^{1,2,3}$, Wei Yu ${ }^{1,2,3} \wedge$
}

${ }^{1}$ Department of Urology, Peking University First Hospital, Beijing, China; ${ }^{2}$ National Urological Cancer Center, Institute of Urology, Peking University, Beijing, China; ${ }^{3}$ Beijing Key Laboratory of Urogenital Diseases (male) Molecular Diagnosis and Treatment Center, Beijing, China

Contributions: (I) Conception and design: W Yu, C Shen; (II) Administrative support: Z He, L Zhou, J Jin; (III) Provision of study materials or patients: W Yu, C Shen, Z He, L Zhou, J Jin; (IV) Collection and assembly of data: J Zhu, Z Liu, Z Zhang, Y Chen, Y Fan; (V) Data analysis and interpretation: J Zhu, Z Liu, Z Zhang; (VI) Manuscript writing: All authors; (VII) Final approval of manuscript: All authors.

\#These authors contributed equally to this work.

Correspondence to: Cheng Shen; Wei Yu. Department of Urology, Peking University First Hospital, No. 8 Xishiku St., Xicheng District, Beijing 100034, China; National Urological Cancer Center, Institute of Urology, Peking University, No. 8 Xishiku St., Xicheng District, Beijing 100034, China; Beijing Key Laboratory of Urogenital Diseases (male) Molecular Diagnosis and Treatment Center, No. 8 Xishiku St., Xicheng District, Beijing 100034, China. Email: shencheng@263.net; yuweif@126.com.

Background: To develop successful prognostic models for grade 4 renal cell carcinoma (RCC) following partial nephrectomy and radical nephrectomy.

Methods: The nomograms were established based on a retrospective study of 135 patients who underwent partial and radical nephrectomy for grade 4 RCC at the Department of Urology, Peking University First Hospital from January 2013 to October 2018. The predictive performance of the nomograms was assessed by the calibration plot and $\mathrm{C}$-index. The results were validated using bootstrap resampling.

Results: Aspartate transaminase (AST), the maximum diameter of tumor (cutoff value $=7 \mathrm{~cm}$ ), lymph node metastasis, and the International Metastatic Renal Cell Carcinoma Database Consortium (IMDC) risk group were independent factors for determining the overall survival (OS) and cancer-specific survival (CSS) in multivariate analysis. AST, the maximum diameter of the tumor (cutoff value $=7 \mathrm{~cm}$ ), and lymph node metastasis were found to be independent variables for progression-free survival (PFS) in multivariate analysis. These variables were used for the studies to establish nomograms. All calibration plots revealed excellent predictive accuracy of the models. The C-indexes of the nomograms for predicting OS, CSS and PFS were 0.729 (95\% CI, 0.659-0.799), 0.725 (95\% CI, 0.654-0.796) and 0.702 (95\% CI, 0.626-0.778), respectively. Moreover, the recurrence rate was not associated with open or laparoscopic radical nephrectomy in our cohort $(\mathrm{P}=0.126)$.

Conclusions: We have developed easy-to-use models that are internally validated to predict postoperative 1-, 3-, and 5-year OS, CSS, and PFS rates of grade 4 RCC patients. The new models could aid in identifying high-risk patients, making postoperative therapeutic and follow-up strategies as well as predicting patients' survival after externally validated. Besides, our study shows that the recurrence rate is not associated with open or laparoscopic radical nephrectomy.

Keywords: Carcinoma; renal cell; nomograms; prognosis; survival analysis

Submitted Mar 01, 2020. Accepted for publication Sep 27, 2020.

doi: $10.21037 /$ tau-19-687

View this article at: http://dx.doi.org/10.21037/tau-19-687

\footnotetext{
^ ORCID: 0000-0002-0938-0308.
} 


\section{Introduction}

Renal cell carcinoma (RCC) is the seventh most common cancer, which represents $2-3 \%$ of all malignant diseases in adults and $90 \%$ of all kidney malignancies $(1,2)$. RCC leads to the tumor-related death of 140,000 patients worldwide every year (3). Fuhrman et al. proposed the nuclear grading system, which has widely been used for grading RCC. Fuhrman grade is one of the most significant prognostic factors for all stages of RCC. The increasing grade represents degrees of biologic aggressiveness and is related to adverse outcomes. Grade 1 is the least aggressive type, while grade 4 is the most $(4,5)$. High Fuhrman grade is associated with increased metastasis rate and decreasing survival rate in patients with RCC $(6,7)$. In a study of 4,063 patients with RCC from eight international institutions, the 10-year cancer-specific survival (CSS) rate of Fuhrman $1-4$ subgroups were $81.0 \%, 56.6 \%, 30.1 \%$, and $18.8 \%$, respectively (8).

Several ways have been identified to distinguish between high-grade and low-grade in RCC. Ding et al. proposed a CT-based model that would make it easier to differentiate high-grade from low-grade clear cell RCC (ccRCC) (9). Kutikov et al. developed a nomogram to identify high-grade RCC using the RENAL Nephrometry Score, which was validated by Wang et al. $(10,11)$. Despite the emergence of targeted therapy, advancement in surgical techniques, and improvements in early diagnosis techniques, the prognosis of high-grade RCC remains poor $(8,12)$. However, there is no tool available to predict the prognosis of patients with grade 4 RCC heretofore.

This study aimed to develop models for predicting the outcomes of Fuhrman grade 4 RCC patients after initial surgery, which is easy to use, comprised of only the most relevant clinicopathological data and capable of accurately predicting the prognosis of grade 4 RCC patients, thus helping urologists with outcome prediction, patient counselling and treatment decisions. We present the following article in accordance with the STROBE reporting checklist (available at http://dx.doi.org/10.21037/tau-20-687).

\section{Methods}

Retrospectively data were collected from patients diagnosed with RCC between January 2013 and October 2018. The study was conducted in accordance with the Declaration of Helsinki (as revised in 2013). And it was approved by institutional ethics board of the Peking University First
Hospital (No.: 2019-241) and individual consent for this retrospective analysis was waived. Out of 4,043 patients, 171 (4.2\%) grade 4 RCC patients were identified based on the final surgical pathology or biopsy samples of the suspicious tissues. All tumor specimens with sarcomatoid or rhabdoid differentiation were classified as grade 4 , according to the International Society of Urological Pathology (ISUP) (13). One hundred and fifty-four patients underwent partial and radical nephrectomy $(\mathrm{RN})$ at our center were included. Nineteen $(12.3 \%)$ patients were failed to follow-up, while 135 patients were included in a multivariate analysis of overall survival (OS). One hundred and thirty-three patients were included in the multivariate analysis of CSS and progression-free survival (PFS) due to the loss of survival data. Pathological confirmation of grade 4 RCC patients, which included in the present analysis, originated from the nephrectomy sample. Pathological variables were derived directly from the pathology reports. Clinical, pathological, and follow-up data were collected and entered into a database.

The following data were recorded for each patient: age at the time of surgery; gender; body mass index (BMI); method of operation; the presence of systemic symptoms (weight loss, fevers, night sweats, etc.); American Joint Commission on Cancer (AJCC) stage; pathological variables, including tumor size, histological subtype, necrosis and so on (based on the 8th edition of the AJCC classification). The International Metastatic Renal Cell Carcinoma Database Consortium (IMDC) score and the Memorial SloanKettering Cancer Center (MSKCC) score both were used for determining the risk stratification. The IMDC score was determined by the official criteria, which are Karnofsky performance status (KPS) $<80 \%$; time from diagnosis to start of systemic treatment $<1$ year; anemia; calcium level; neutrophils; platelets greater than the upper normal limit (UNL). The MSKCC score was calculated using the official criteria: lactate dehydrogenase (LDH) level >1.5 UNL; KPS $<80 \%$; time from diagnosis to start the systemic treatment $<1$ year; anemia; calcium level higher than the UNL. All patients were assigned to the intermediate and poor groups since no patient received systemic treatment one year after the diagnosis. Other recorded variables were performed, including blood test and biochemical indices, prognostic parameters, treatment modalities, and survival data. We used descriptive statistics to characterize the patient sample. OS was calculated based on the time of surgery to the time of death or the last follow-up while CSS was derived from the date of RCC surgery to the death or the last follow-up. 
However, PFS was calculated from the time of surgery to the time of disease progression, death, or the last follow-up.

\section{Statistical analysis}

Univariate and multivariate analyses of OS, PFS, CSS were performed to identify prognostic factors using Cox regression analysis, while hazard ratio (HR) was used to measure the effect of these factors. The survival curves have been estimated by the Kaplan-Meier method, while nomograms were studied to predict the postoperative (1-, 3 -, and 5-year) survival using the multivariate analysis. The predictive ability was assessed using the C-index presented by Harrell et al. (14). Both Bootstrap validation and calibration plot were used to perform the internal validation of 200 samples. Analysis of the relationship between operative types and recurrence rate was conducted by the chi-square test. $\mathrm{R}$ software (version 3.5.2) using "rms" package was used to calculate the nomograms and calibration curves. SPSS 22.0 (IBM Corp., Armonk, NY, USA) was used to analyze the other statistical calculations, and a $P$ value $<0.05$ was considered as statistically significant.

\section{Results}

Table 1 presents demographic and clinicopathological

Table 1 Clinicopathologic features of grade 4 RCC patients

\begin{tabular}{lc}
\hline Characteristic & Value $(\mathrm{n}=154)$ \\
\hline Median age at diagnosis [range], years & $58[19-83]$ \\
Mean OS $(95 \% \mathrm{Cl})$, months & $46.2(40.742-51.651)$ \\
Mean CSS (95\% Cl), months & $45.8(41.317-52.278)$ \\
Mean PFS (95\% Cl), months & $46.8(40.010-51.518)$ \\
Mean follow-up [IQR], months & $35.8[27-48]$ \\
Sex, n (\%) & $107(69.5)$ \\
Male & $47(30.5)$ \\
Female & \\
BMI, kg/m ${ }^{2}, \mathrm{n}(\%)$ & $10(6.5)$ \\
$<18.5$ & $67(43.5)$ \\
$18.5-23.9$ & $61(39.6)$ \\
$24.0-27.9$ & \\
\hline
\end{tabular}

Table 1 (continued)
Table 1 (continued)

\begin{tabular}{|c|c|}
\hline Characteristic & Value $(n=154)$ \\
\hline$\geq 28.0$ & $16(10.4)$ \\
\hline \multicolumn{2}{|c|}{ Side of nephrectomy, n (\%) } \\
\hline Left & $70(45.5)$ \\
\hline Right & $84(54.5)$ \\
\hline \multicolumn{2}{|c|}{ Systemic symptoms, n (\%) } \\
\hline No & $61(39.6)$ \\
\hline Yes & $93(60.4)$ \\
\hline \multicolumn{2}{|c|}{ Primary tumor size, n (\%) } \\
\hline T1a & $7(4.5)$ \\
\hline $\mathrm{T} 1 \mathrm{~b}$ & $18(11.7)$ \\
\hline $\mathrm{T} 2 \mathrm{a}$ & $7(4.5)$ \\
\hline $\mathrm{T} 2 \mathrm{~b}$ & $7(4.5)$ \\
\hline Т3a & $79(51.3)$ \\
\hline T3b & $13(8.4)$ \\
\hline T3c & $2(1.3)$ \\
\hline $\mathrm{T} 4$ & $21(13.6)$ \\
\hline \multicolumn{2}{|c|}{ Primary tumor nodal involvement, $\mathrm{n}(\%)$} \\
\hline NO & $120(77.9)$ \\
\hline $\mathrm{N} 1$ & $33(21.4)$ \\
\hline NX & $1(0.6)$ \\
\hline \multicolumn{2}{|c|}{ Initial metastatic status, n (\%) } \\
\hline MO at diagnosis & $119(77.3)$ \\
\hline M1 at diagnosis & $35(22.7)$ \\
\hline \multicolumn{2}{|l|}{ AJCC stage, $\mathrm{n}(\%)$} \\
\hline 1 & $20(13.0)$ \\
\hline II & $12(7.8)$ \\
\hline III & $74(48.1)$ \\
\hline IV & $48(31.2)$ \\
\hline \multicolumn{2}{|c|}{ Adrenal invasion, $\mathrm{n}(\%)$} \\
\hline No & $142(92.2)$ \\
\hline Yes & $12(7.8)$ \\
\hline \multicolumn{2}{|c|}{ Vascular invasion, n (\%) } \\
\hline No & $81(52.6)$ \\
\hline Yes & $73(47.4)$ \\
\hline
\end{tabular}

Table 1 (continued) 
Table 1 (continued)

\begin{tabular}{|c|c|}
\hline Characteristic & Value $(n=154)$ \\
\hline \multicolumn{2}{|l|}{ Histological subtype, n (\%) } \\
\hline Clear cell & $110(71.4)$ \\
\hline Papillary & $7(4.5)$ \\
\hline Clear cell/papillary & $16(10.4)$ \\
\hline Unclassified & $17(11.0)$ \\
\hline Collecting type & $4(2.6)$ \\
\hline \multicolumn{2}{|l|}{ Sarcomatoid differentiation, $\mathrm{n}(\%)$} \\
\hline No & $53(34.4)$ \\
\hline Yes & $101(65.6)$ \\
\hline \multicolumn{2}{|l|}{ Rhabdoid differentiation, n (\%) } \\
\hline No & $119(77.3)$ \\
\hline Yes & $35(22.7)$ \\
\hline \multicolumn{2}{|l|}{ Cancer embolus, n (\%) } \\
\hline No & $102(66.2)$ \\
\hline Yes & $52(33.8)$ \\
\hline \multicolumn{2}{|l|}{ Necrosis, n (\%) } \\
\hline No & $52(33.8)$ \\
\hline Yes & $102(66.2)$ \\
\hline \multicolumn{2}{|c|}{ Time from initial diagnosis to start of systemic therapy } \\
\hline$<12$ months & $146(94.8)$ \\
\hline$>12$ months & $8(5.2)$ \\
\hline \multicolumn{2}{|l|}{ Karnofsky index, n (\%) } \\
\hline$>80 \%$ & $133(86.4)$ \\
\hline$<80 \%$ & $21(13.6)$ \\
\hline \multicolumn{2}{|c|}{ Median baseline laboratory parameters (range) } \\
\hline Haemoglobin, g/dL & $11.5-15.0$ \\
\hline Neutrophils, per $\mathrm{mm}^{3}$ & $1,800-6,300$ \\
\hline Platelets, G/L & $125-350$ \\
\hline $\mathrm{LDH}, \mathrm{IU} / \mathrm{L}$ & $100-240$ \\
\hline Corrected calcium, mmol/L & $2.12-2.75$ \\
\hline Lymphocytes, per $\mathrm{mm}^{3}$ & $1,100-3,200$ \\
\hline Albumin before nephrectomy, $\mathrm{g} / \mathrm{L}$ & $40-55$ \\
\hline \multicolumn{2}{|l|}{ Site of recurrence, $\mathrm{n}(\%)$} \\
\hline In situ & $2(1.3)$ \\
\hline Lymph node & $1(0.6)$ \\
\hline
\end{tabular}

Table 1 (continued)
Table 1 (continued)

\begin{tabular}{lc}
\hline Characteristic & Value $(\mathrm{n}=154)$ \\
\hline Metastasis & $30(19.5)$ \\
Unknown & $5(3.2)$ \\
IMDC risk group, $\mathrm{n}(\%)$ & \\
Intermediate & $98(63.6)$ \\
Poor & $56(36.4)$ \\
MSKCC risk group, $\mathrm{n}(\%)$ & \\
Intermediate & $77(50.0)$ \\
Poor & $11(7.1)$ \\
Unknown & $66(42.9)$ \\
\hline
\end{tabular}

§, loss of clinical or follow-up data. AST, aspartate aminotransferase; FIB, fibrinogen; HR, hazard ratio; Cl, confidence interval; LN, lymph node; RCC, renal cell carcinoma. IMDC, the International Metastatic Renal Cell Carcinoma Database Consortium; MSKCC, the Memorial Sloan Kettering Cancer Center; LDH, lactate dehydrogenase; IQR, interquartile range.

characteristics. The present study included 154 grade 4 RCC patients who underwent partial and $\mathrm{RN}$ at our center. The median age was 58 years (range, 19-83 years). After a mean follow-up time of 35.8 months [interquartile range (IQR), 27-48 months], 51/135 (37.8\%) patients had died from various causes. The mean OS, CSS and PFS are 46.2 (95\% CI, 40.742-51.651); 46.8 (95\% CI, 40.010-51.518); 45.8 (95\% CI, 41.317-52.278) months, respectively (please see Figure 1). Treatment modalities, as well as detailed adjunctive therapy and response of grade 4 RCC patients in our cohort, are listed in supplementary files (Tables S1,S2). One hundred and forty-eight $(96.1 \%)$ patients underwent $\mathrm{RN}$, while only 6 (3.9\%) patients received laparoscopic partial nephrectomy. Besides, the recurrence rate was not associated with open or laparoscopic $\mathrm{RN}(\mathrm{P}=0.126)$.

Results from the univariate analysis showed that aspartate transaminase (AST), fibrinogen (FIB), the maximum diameter of tumor, targeted therapy, systemic symptom, adrenal invasion, metastasis (bone and visceral metastasis), lymph node (LN) metastasis, and IMDC risk group all were prognostic factors of OS and CSS, as shown in Tables 2,3. In the univariate analysis, sarcomatoid differentiation, and the above nine variables were significant for PFS, as presented in Table 4. The above-related parameters were then included in multivariate Cox proportional hazards analysis. Besides, we identified four variables, which are AST, the maximum diameter of tumor (cutoff value $=7 \mathrm{~cm}$ ), 

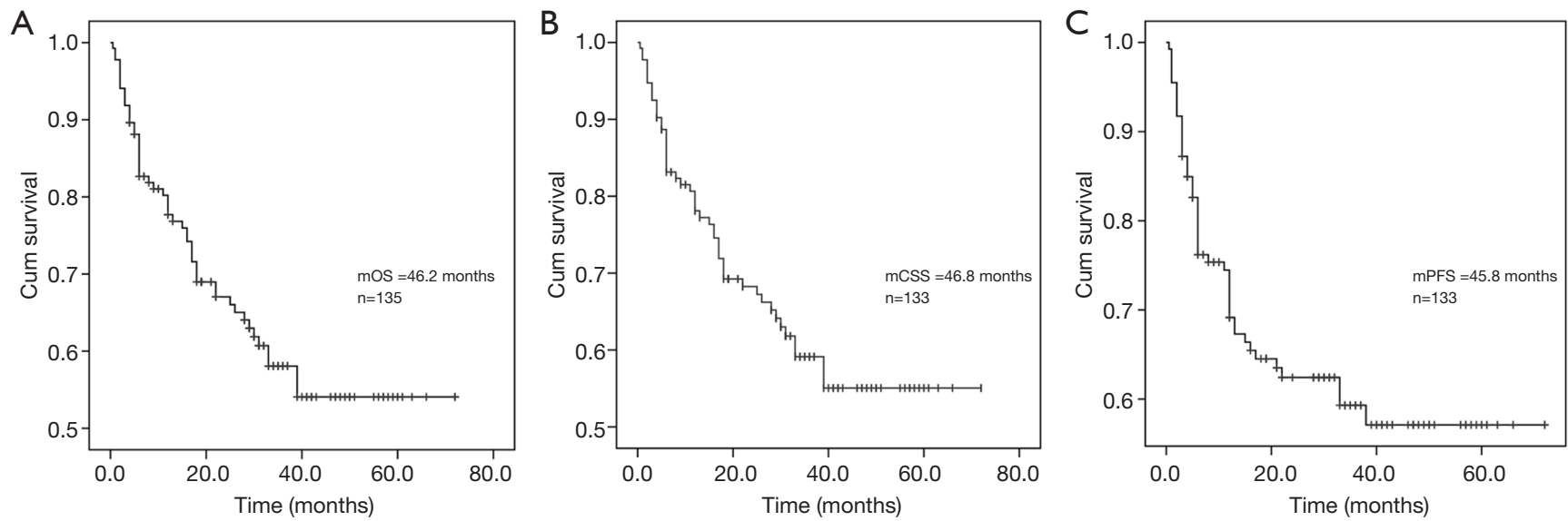

Figure 1 Kaplan-Meier plots for overall survival (A), cancer-specific survival (B), and progression-free survival (C) in patients with grade 4 RCC. mOS, mean overall survival; mCSS, mean cancer-specific survival; mPFS, median progression-free survival; RCC, renal cell carcinoma. Time was measured in months.

Table 2 Univariate and multivariate analyses of predictors of OS

\begin{tabular}{|c|c|c|c|c|}
\hline Characteristic & \multicolumn{2}{|c|}{ Univariate Cox } & \multicolumn{2}{|c|}{ Multivariate Cox } \\
\hline AST & $2.155(1.220-3.805)$ & 0.008 & 2.539 (1.390-4.638) & 0.002 \\
\hline FIB & $2.549(1.375-4.723)$ & 0.003 & - & 0.290 \\
\hline Systemic symptom & $1.885(1.043-3.409)$ & 0.036 & - & 0.545 \\
\hline Tumor size & $2.293(1.267-4.150)$ & 0.006 & $2.073(1.131-3.801)$ & 0.018 \\
\hline Targeted therapy & $1.881(1.081-3.275)$ & 0.025 & - & 0.432 \\
\hline Sarcomatous differentiation & $1.808(0.963-3.398)$ & 0.066 & - & - \\
\hline Rhabdoid differentiation & $0.545(0256-1.160)$ & 0.115 & - & - \\
\hline RCC/non-RCC & $1.061(0.573-1.965)$ & 0.851 & - & - \\
\hline Tumor thrombus & $1.238(0.702-2.186)$ & 0.461 & - & - \\
\hline Vascular invasion & $1.055(0.609-1.830)$ & 0.848 & - & - \\
\hline Necrosis & $0.977(0.545-1.750)$ & 0.938 & - & - \\
\hline IMDC risk group & $2.011(1.159-3.488)$ & 0.013 & $1.781(1.006-3.151)$ & 0.048 \\
\hline MSKCC risk group & $0.803(0.190-3.405)$ & 0.766 & - & - \\
\hline
\end{tabular}

AST, aspartate aminotransferase; FIB, fibrinogen; HR, hazard ratio; Cl, confidence interval; LN, lymph node; RCC, renal cell carcinoma. IMDC, the International Metastatic Renal Cell Carcinoma Database Consortium; MSKCC, the Memorial Sloan Kettering Cancer Center. 
Table 3 Univariate and multivariate analyses of predictors of CSS

\begin{tabular}{|c|c|c|c|c|}
\hline Characteristic & \multicolumn{2}{|c|}{ Univariate Cox } & \multicolumn{2}{|c|}{ Multivariate Cox } \\
\hline AST & $2.013(1.175-3.763)$ & 0.012 & $2.498(1.349-4.626)$ & 0.004 \\
\hline FIB & $2.454(1.318-4.569)$ & 0.005 & - & 0.347 \\
\hline Systemic symptom & $1.812(0.996-3.293)$ & 0.051 & - & 0.639 \\
\hline Tumor size & $2.201(1.209-4.006)$ & 0.010 & $1.987(1.077-3.663)$ & 0.028 \\
\hline Targeted therapy & $1.849(1.050-3.257)$ & 0.033 & - & 0.426 \\
\hline Sarcomatous differentiation & $1.870(0.974-3.590)$ & 0.060 & - & - \\
\hline Rhabdoid differentiation & $0.565(0.265-1.206)$ & 1.140 & - & - \\
\hline RCC/non-RCC & $1.042(0.552-1.968)$ & 0.898 & - & - \\
\hline Tumor thrombus & $1.307(0.735-2.323)$ & 0.362 & - & - \\
\hline Vascular invasion & $1.122(0.641-1.966)$ & 0.687 & - & - \\
\hline Necrosis & $1.014(0.558-1.844)$ & 0.964 & - & - \\
\hline IMDC risk group & $1.991(1.135-3.493)$ & 0.016 & $1.791(1.002-3.202)$ & 0.049 \\
\hline MSKCC risk group & $0.834(0.196-3.543)$ & 0.806 & - & - \\
\hline
\end{tabular}

AST, aspartate aminotransferase; FIB, fibrinogen; HR, hazard ratio; Cl, confidence interval; LN, lymph node; RCC, renal cell carcinoma. IMDC, the International Metastatic Renal Cell Carcinoma Database Consortium; MSKCC, the Memorial Sloan Kettering Cancer Center.

LN metastasis, and IMDC risk group, as independent prognostic variables for OS and CSS (Tables 2,3). Further, the first three variables mentioned above were independent prognostic factors for PFS, as shown in Table 4. Figures 2-4 depict the prognostic models that were established based on all the independent variables to accurately predict the OS, CSS, and PFS of patients with grade 4 RCC after surgery. The nomograms, which were developed based on the present analysis, can predict 1-, 3-, and 5-year postoperative OS, CSS, and PFS for grade 4 RCC patients following initial surgery. Figure 5 presents the internal calibration curves of the nomograms, indicating that the models were reasonably accurate. Moreover, the $\mathrm{C}$-indexes of the multivariate prognostic model for OS, CSS and PFS were found to be 0.729 (95\% CI, 0.659-0.799), 0.725 (95\% CI, $0.654-0.796)$ and 0.702 (95\% CI, 0.626-0.778), respectively.

\section{Discussion}

Fuhrman grading system is one of the most significant prognostic factors for all stages of RCC $(4,5)$. The high nuclear grade is related to increasing metastasis rates and reduced disease-free survival $(6,7,15)$. Several studies have proposed to distinguish high-grade from low-grade RCC and identified a few prognostic factors. Ding et al. proposed a CT-based model to facilitate discriminating the highgrade from low-grade ccRCC (9). Kutikov et al. developed a nomogram to identify high-grade RCC using the RENAL nephrometry score (10). Kara et al. found that sarcomatoid differentiation had independently predicted worse survival in grade 4 RCC patients (16). However, there is currently no model for the prediction of survival of patients with grade 4 RCC after the initial surgery. With this in mind, we have tried to establish a predictive nomogram based on clinicopathological and preoperative laboratory variables to evaluate the postoperative survival rate of patients with grade 4 RCC.

Multivariate analysis showed the parameters which can independently predict postoperative OS, CSS, and PFS. The maximum diameter of the tumor (cutoff value $=7 \mathrm{~cm}$ ), 
Table 4 Univariate and multivariate analyses of predictors of PFS

\begin{tabular}{|c|c|c|c|c|}
\hline Characteristic & \multicolumn{2}{|c|}{ Univariate Cox } & \multicolumn{2}{|c|}{ Multivariate Cox } \\
\hline AST & $2.237(1.258-3.976)$ & 0.006 & $2.790(1.545-5.036)$ & 0.001 \\
\hline FIB & $2.271(1.220-4.226)$ & 0.010 & - & 0.182 \\
\hline Systemic symptom & $1.829(1.006-3.327)$ & 0.048 & - & 0.238 \\
\hline Tumor size & $2.151(1.183-3.913)$ & 0.012 & $2.142(1.153-3.980)$ & 0.016 \\
\hline Targeted therapy & $1.915(1.087-3.371)$ & 0.024 & - & 0.338 \\
\hline Sarcomatous differentiation & $2.024(1.034-3.961)$ & 0.040 & - & 0.112 \\
\hline Rhabdoid differentiation & $0.585(0.274-1.249)$ & 0.166 & - & - \\
\hline RCC/non-RCC & $1.081(0.573-2.039)$ & 0.810 & - & - \\
\hline Tumor thrombus & $1.258(0.704-2.249)$ & 0.439 & - & - \\
\hline Vascular invasion & $1.106(0.631-1.938)$ & 0.726 & - & - \\
\hline Necrosis & $1.033(0.568-1.878)$ & 0.915 & - & - \\
\hline IMDC risk group & $1.914(1.091-3.488)$ & 0.024 & - & 0.090 \\
\hline MSKCC risk group & $1.081(0.573-2.039)$ & 0.810 & - & - \\
\hline
\end{tabular}

AST, aspartate aminotransferase; FIB, fibrinogen; HR, hazard ratio; Cl, confidence interval; LN, lymph node; RCC, renal cell carcinoma. IMDC, the International Metastatic Renal Cell Carcinoma Database Consortium; MSKCC, the Memorial Sloan Kettering Cancer Center.

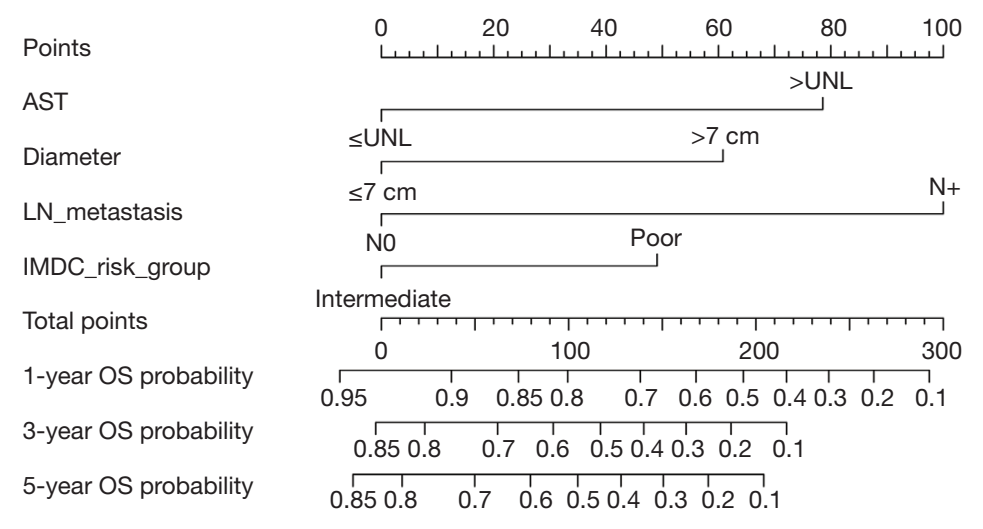

Figure 2 Nomogram to predict postoperative overall survival (OS) for grade 4 RCC patients. To use the nomogram, first, allocate the points of each variable of the patient by drawing a line straight upward to the point axis, then, attach all the points, and draw a line straight down from the total points axis to get the 1-, 3-, and 5-year OS to obtain the probability of survival. AST, aspartate transaminase; UNL, upper normal limit; LN, lymph node; IMDC, International Metastatic Renal Cell Carcinoma Database Consortium; RCC, renal cell carcinoma. 


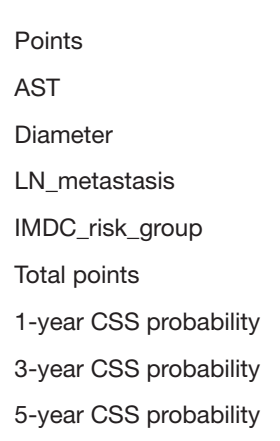

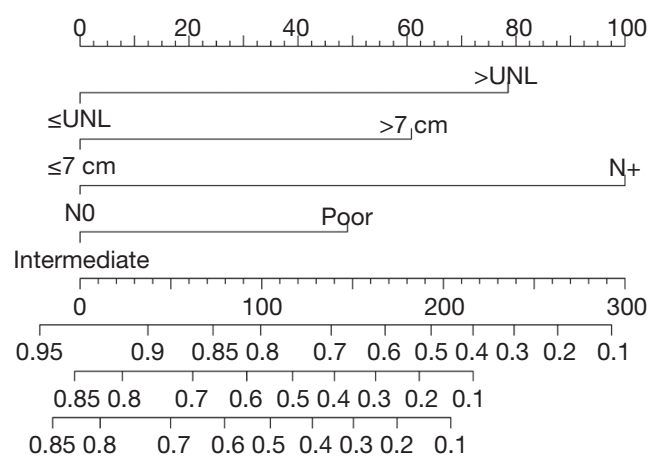

Figure 3 Nomogram for prediction of postoperative cancer-specific survival (CSS) in patients with grade 4 RCC. To use the nomogram, first, assign the points of each variable of the patient by drawing a line straight up to the point axis, then, add all the points, and draw a line straight down from the total point axis to obtain the 1-, 3-, and 5-year CSS for the probability of survival. AST, aspartate transaminase; UNL, upper normal limit; LN, lymph node; IMDC, International Metastatic Renal Cell Carcinoma Database Consortium; RCC, renal cell carcinoma.

Points
AST
Diameter
LN_metastasis
IMDC_risk_group
Total points
1-year PFS probability
3-year PFS probability
5-year PFS probability

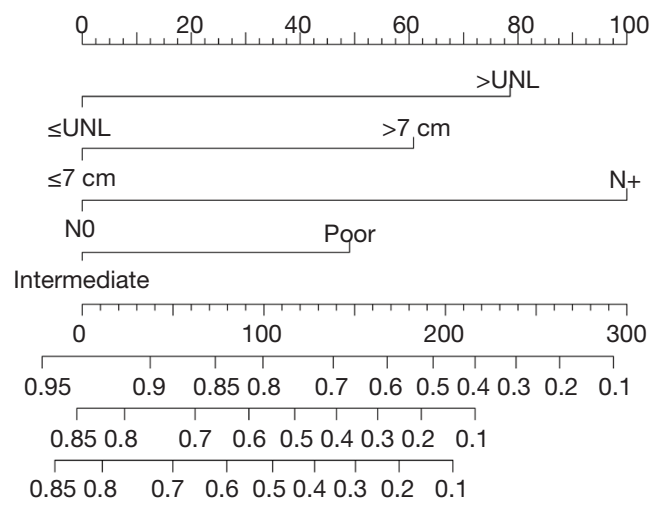

Figure 4 Nomogram for the prediction of postoperative progression-free survival (PFS) in patients with grade 4 RCC. To use the nomogram, first, assign the points of each variable of the patient by drawing a line straight up to the point axis, then, add all the points, and draw a line straight down from the total point axis to obtain the 1-, 3-, and 5-year PFS for the probability of survival. AST, aspartate transaminase; UNL, upper normal limit; LN, lymph node; IMDC, International Metastatic Renal Cell Carcinoma Database Consortium; RCC, renal cell carcinoma.

LN metastasis, IMDC risk group, and AST are commonly available variables that can easily be obtained from clinical data. Based on the four variables above, clinical models that were developed to predict the probability of postoperative 1-, 3-, and 5-year OS, CSS, and PFS in grade 4 RCC patients (Figures 2-4). Calibration curves of the nomograms performed well in predicting OS, CSS, and PFS compared with the ideal model, as shown in Figure 5. The C-indexes also manifested that the nomograms could well predict postoperative OS, CSS, and PFS of patients with grade 4 RCC. Therefore, patients with high-risk disease should receive more active surveillance and consider participating in clinical trials.

It is to be noted that both IMDC and MSKCC scores have been used to predict the prognosis of metastatic RCC (mRCC) $(17,18)$. In the report, we explored the first-time prognostic value of IMDC and MSKCC score in grade 4 RCC, considering grade 4 RCC is more prone to metastasis than the other three types. Univariate and multivariate analyses indicated that the IMDC risk group was associated with OS and CSS of grade 4 RCC while MSKCC score not (Tables 2,3). And the models performed well with the IMDC 
A
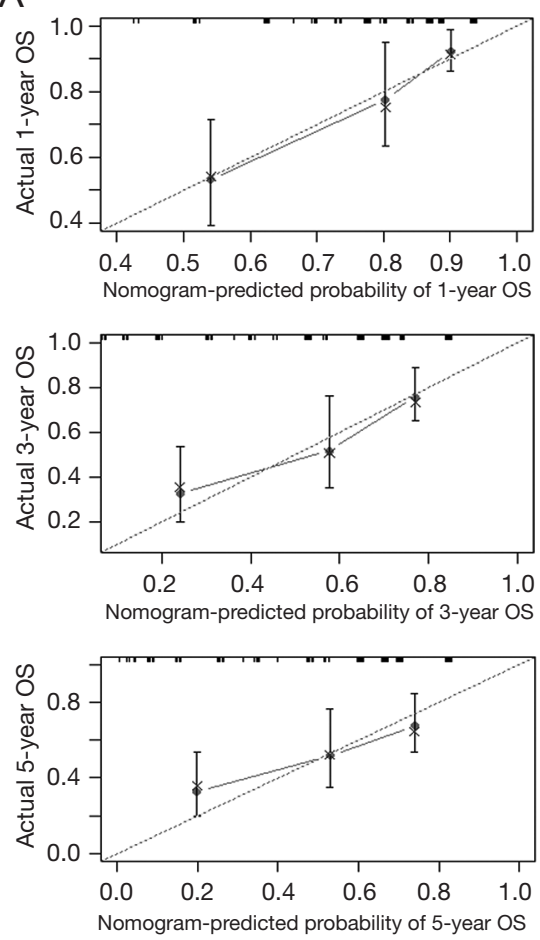

B
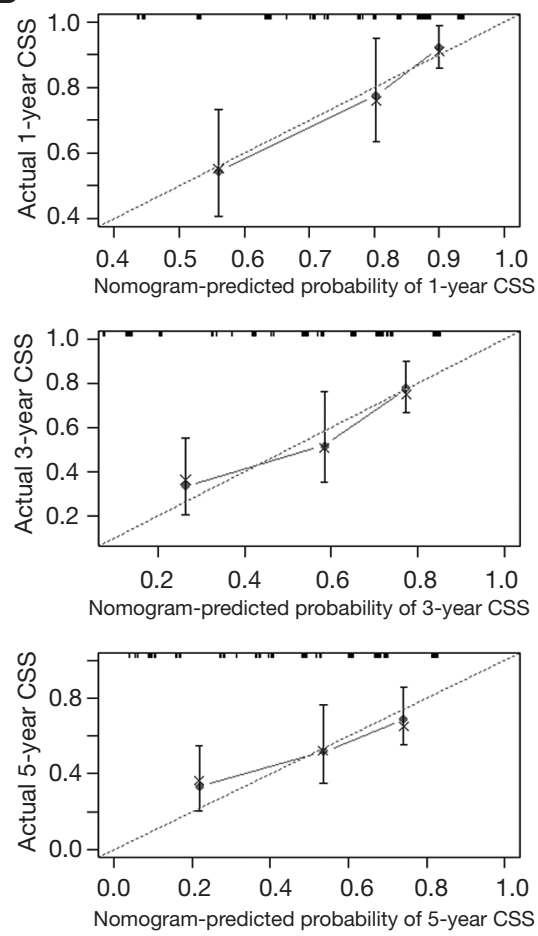

C
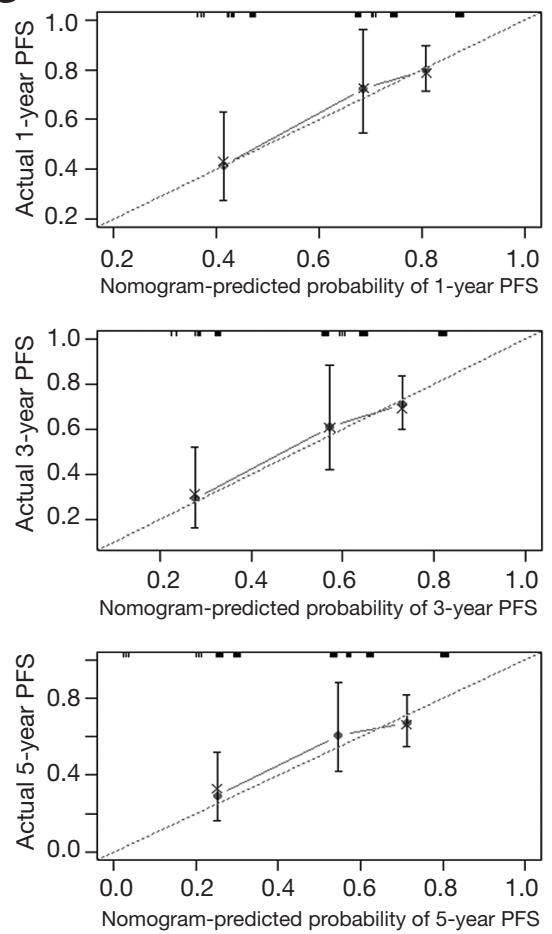

Figure 5 The internal calibration plots for prediction of overall survival (A), cancer-specific survival (B), and progression-free survival (C) at 1,3, and 5 years postoperatively. The dotted lines represent the perfect match between nomogram-predicted probability and the actual probability. Black lines show the performance of the proposed models. Black dots are sub-groups of the present data sets; X represents a bootstrapped corrected estimation of the models. Vertical bars represent $95 \%$ CI.

risk group included.

In this context, a previous report by Suer et al. found that tumor size was related to the outcome of patients with RCC, i.e., pT3a RCC larger than $7 \mathrm{~cm}$ showed a worse result (19). Chen et al. also concluded that the maximum tumor diameter had a significant impact on the outcome in patients with pT3aN0M0 RCC and a 7-cm cutoff could help in improving the prognostic discrimination (20). According to the AJCC 2016 TNM system, a cutoff of $7 \mathrm{~cm}$ is solely used to differentiate stage T1 and T2 in the RCC. Therefore, it is reasonable that we set $7 \mathrm{~cm}$ as the cutoff value for tumor size, and it turned out to be an independent prognostic factor of grade 4 RCC.

Bezan et al. first found that the AST/alanine aminotransferase (ALT) ratio (De Ritis ratio) could be a prognostic factor for metastasis-free survival and OS in localized RCC (21). Lee et al. suggested that its prognostic value should be limited to ccRCC (22). In our cohort, the De Ritis ratio was not significant in the univariate analysis, given the presence of metastatic and non-ccRCC patients, which is reasonable. On the other hand, we explored the prognostic value of AST and demonstrated that AST was an independent prognostic factor of grade 4 RCC for the first time.

LN metastasis was an independent predictor of inferior outcome according to previous study. Zubac et al. underlined that the status of the LNs was independently associated with CSS after the RN (23). Besides, Pantuck et al. reported that isolated $\mathrm{LN}$ was associated with a poor prognosis of RN outcomes (24). In our study, LN metastasis was significant in both univariate and multivariate analysis for OS, PFS, and CSS (Tables 2-4), which were consistent with previous studies.

Several studies have explored the outcome of RN and nephron-sparing surgery (NSS) for certain types and sizes of RCC (25-27). However, no research has focused on the association between operative types and outcomes of grade 4 RCC. For the first time, we found that the recurrence rate was not associated with open or laparoscopic $\mathrm{RN}(\mathrm{P}=0.126)$. As far as adjuvant therapy is concerned, several metaanalyses revealed the effects of radiotherapy, chemotherapy, 
and immunotherapy. Tunio et al. have shown that adjuvant radiotherapy does not affect OS and disease-free survival (28). Bai et al. confirmed that immune therapy was not useful for RCC (29) patients. Besides, RCC has traditionally been categorized as resistant to chemotherapy (30). Therefore, very few patients received these adjuvant therapies in our cohort.

It should be acknowledged that there are some limitations to our study. Nomograms were established based on the data from a single institution over a relatively short period, and the sample size was also small. Besides, the retrospective nature of our study may have caused inherent structural biases, and the follow-up time was relatively short. Third, some important prognostic factors such as surgical methods, immunotherapy, chemotherapy, and radiotherapy have not been included in the analyses, as very few patients in our cohort have received these treatments. Finally, external validation by long-term follow-up is required.

In conclusion, the present study has proved that AST, the maximum diameter of tumor (cutoff value $=7 \mathrm{~cm}$ ), $\mathrm{LN}$ metastasis, and IMDC risk group are independent risk variables for post-operative survival of grade 4 RCC patients. Accurate nomograms based on these variables are currently the only tools available to predict the 1-, 3 -, and 5-year postoperative OS, CSS, and PFS rates of grade 4 RCC patients. These models could help to identify urologically high-risk patients, develop postoperative therapeutic and follow-up strategies, and to predict survival after external validation. Moreover, the recurrence rate is not associated with an open or laparoscopic RN.

\section{Acknowledgments}

Funding: None.

\section{Footnote}

Reporting Checklist: The authors have completed the STROBE reporting checklist. Available at http://dx.doi. org/10.21037/tau-20-687

Data Sharing Statement: Available at http://dx.doi. org/10.21037/tau-20-687

Peer Review File: Available at http://dx.doi.org/10.21037/ tau-20-687

Conflicts of Interest: All authors have completed the ICMJE uniform disclosure form (available at http://dx.doi. org/10.21037/tau-20-687). The authors have no conflicts of interest to declare.

Ethical Statement: The authors are accountable for all aspects of the work in ensuring that questions related to the accuracy or integrity of any part of the work are appropriately investigated and resolved. The study was conducted in accordance with the Declaration of Helsinki (as revised in 2013). And it was approved by institutional ethics board of the Peking University First Hospital (No.: 2019241) and individual consent for this retrospective analysis was waived.

Open Access Statement: This is an Open Access article distributed in accordance with the Creative Commons Attribution-NonCommercial-NoDerivs 4.0 International License (CC BY-NC-ND 4.0), which permits the noncommercial replication and distribution of the article with the strict proviso that no changes or edits are made and the original work is properly cited (including links to both the formal publication through the relevant DOI and the license). See: https://creativecommons.org/licenses/by-nc-nd/4.0/.

\section{References}

1. Gupta K, Miller JD, Li JZ, et al. Epidemiologic and socioeconomic burden of metastatic renal cell carcinoma (mRCC): a literature review. Cancer Treat Rev 2008;34:193-205.

2. Znaor A, Lortet-Tieulent J, Laversanne M, et al. International variations and trends in renal cell carcinoma incidence and mortality. Eur Urol 2015;67:519-30.

3. Capitanio U, Montorsi F. Renal cancer. Lancet 2016;387:894-906.

4. Fuhrman SA, Lasky LC, Limas C. Prognostic significance of morphologic parameters in renal cell carcinoma. Am J Surg Pathol 1982;6:655-63.

5. Cheville JC, Blute ML, Zincke H, et al. Stage pT1 conventional (clear cell) renal cell carcinmoa: pathological features associated with cancer specific survival. J Urol 2001;166:453-6.

6. Smith ZL, Pietzak EJ, Meise CK, et al. Simplification of the Fuhrman grading system for renal cell carcinoma. Can J Urol 2015;22:8069-73.

7. Zisman A, Pantuck AJ, Dorey F, et al. Improved prognostication of renal cell carcinoma using an integrated staging system. J Clin Oncol 2001;19:1649-57. 
8. Patard JJ, Leray E, Rioux-Leclercq N, et al. Prognostic value of histologic subtypes in renal cell carcinoma: a multicenter experience. J Clin Oncol 2005;23:2763-71.

9. Ding J, Xing Z, Jiang Z, et al. CT-based radiomic model predicts high grade of clear cell renal cell carcinoma. Eur J Radiol 2018;103:51-6.

10. Kutikov A, Smaldone MC, Egleston BL, et al. Anatomic features of enhancing renal masses predict malignant and high-grade pathology: a preoperative nomogram using the RENAL Nephrometry score. Eur Urol 2011;60:241-8.

11. Wang HK, Zhu Y, Yao XD, et al. External validation of a nomogram using RENAL nephrometry score to predict high grade renal cell carcinoma. J Urol 2012;187:1555-60.

12. Lane BR, Babineau D, Kattan MW, et al. A preoperative prognostic nomogram for solid enhancing renal tumors $7 \mathrm{~cm}$ or less amenable to partial nephrectomy. J Urol 2007;178:429-34.

13. Delahunt B, Cheville JC, Martignoni G, et al. The International Society of Urological Pathology (ISUP) grading system for renal cell carcinoma and other prognostic parameters. Am J Surg Pathol 2013;37:1490-504.

14. Harrell FE Jr, Lee KL, Mark DB. Multivariable prognostic models: issues in developing models, evaluating assumptions and adequacy, and measuring and reducing errors. Stat Med 1996;15:361-87.

15. Griffiths DF, Verghese A, Golash A, et al. Contribution of grade, vascular invasion and age to outcome in clinically localized renal cell carcinoma. BJU Int 2002;90:26-31.

16. Kara O, Maurice MJ, Zargar H, et al. Prognostic implications of sarcomatoid and rhabdoid differentiation in patients with grade 4 renal cell carcinoma. Int Urol Nephrol 2016;48:1253-60.

17. Motzer RJ, Bacik J, Murphy BA, et al. Interferon-alfa as a comparative treatment for clinical trials of new therapies against advanced renal cell carcinoma. J Clin Oncol 2002;20:289-96.

18. Heng DY, Xie W, Regan MM, et al. Prognostic factors for overall survival in patients with metastatic renal cell carcinoma treated with vascular endothelial growth factortargeted agents: results from a large, multicenter study. J Clin Oncol 2009;27:5794-9.

19. Süer E, Baltaci S, Burgu B, et al. Significance of tumor size in renal cell cancer with perinephric fat infiltration: is TNM staging system adequate for predicting prognosis? Urol J 2013;10:774-9.

20. Chen L, Ma X, Li H, et al. Influence of tumor size on oncological outcomes of pathological T3aN0M0 renal cell carcinoma treated by radical nephrectomy. PLoS One 2017;12:e0173953.

21. Bezan A, Mrsic E, Krieger D, et al. The Preoperative AST/ALT (De Ritis) Ratio Represents a Poor Prognostic Factor in a Cohort of Patients with Nonmetastatic Renal Cell Carcinoma. J Urol 2015;194:30-5.

22. Lee H, Lee SE, Byun SS, et al. De Ritis ratio (aspartate transaminase/alanine transaminase ratio) as a significant prognostic factor after surgical treatment in patients with clear-cell localized renal cell carcinoma: a propensity score-matched study. BJU Int 2017;119:261-7.

23. Zubac DP, Bostad L, Seidal T, et al. The prognostic relevance of interactions between venous invasion, lymph node involvement and distant metastases in renal cell carcinoma after radical nephrectomy. BMC Urol 2008;8:19.

24. Pantuck AJ, Zisman A, Dorey F, et al. Renal cell carcinoma with retroperitoneal lymph nodes: role of lymph node dissection. J Urol 2003;169:2076-83.

25. Kalogirou C, Fender H, Muck P, et al. Long-Term Outcome of Nephron-Sparing Surgery Compared to Radical Nephrectomy for Renal Cell Carcinoma $\geq 4 \mathrm{~cm}$ - A Matched-Pair Single Institution Analysis. Urol Int 2017;98:138-47.

26. Koo KC, Kim JC, Cho KS, et al. Oncological outcomes after partial vs radical nephrectomy in renal cell carcinomas of $\leq 7 \mathrm{~cm}$ with presumed renal sinus fat invasion on preoperative imaging. BJU Int 2016;117:87-93.

27. Venkatramani V, Koru-Sengul T, Miao F, et al. A comparison of overall survival and perioperative outcomes between partial and radical nephrectomy for $\mathrm{cT} 1 \mathrm{~b}$ and cT2 renal cell carcinoma-Analysis of a national cancer registry. Urol Oncol 2018;36:90.e9-90.e14.

28. Tunio MA, Hashmi A, Rafi M. Need for a new trial to evaluate postoperative radiotherapy in renal cell carcinoma: a meta-analysis of randomized controlled trials. Ann Oncol 2010;21:1839-45.

29. Bai Y, Li S, Jia Z, et al. Adjuvant therapy for locally advanced renal cell carcinoma: A meta-analysis and systematic review. Urol Oncol 2018;36:79.e1-79.e10.

30. Buti S, Bersanelli M, Sikokis A, et al. Chemotherapy in metastatic renal cell carcinoma today? A systematic review. Anticancer Drugs 2013;24:535-54.

Cite this article as: Zhu J, Liu Z, Zhang Z, Fan Y, Chen Y, He Z, Zhou L, Jin J, Shen C, Yu W. Development and internal validation of nomograms for the prediction of postoperative survival of patients with grade 4 renal cell carcinoma (RCC). Transl Androl Urol 2020;9(6):2629-2639. doi: 10.21037/tau-19-687 
Supplementary

Table S1 Modalities of treatment in patients with grade 4 RCC

\begin{tabular}{|c|c|}
\hline Characteristic & Value \\
\hline \multicolumn{2}{|l|}{ Laparotomy } \\
\hline Nephrectomy & $67(43.5)$ \\
\hline \multicolumn{2}{|l|}{ Laparoscopy } \\
\hline Partial nephrectomy & $6(3.9)$ \\
\hline \multicolumn{2}{|c|}{ Targeted therapies $(n=46), n(\%)$} \\
\hline Sunitinib & $11(7.1)$ \\
\hline Sorafenib & $11(7.1)$ \\
\hline Sorafenib + axitinib & $1(0.6)$ \\
\hline Unknown ${ }^{\S}$ & $20(13.0)$ \\
\hline \multicolumn{2}{|c|}{ Immunotherapy (n=2), n (\%) } \\
\hline Interferon $\alpha$ & $2(1.3)$ \\
\hline Radiotherapy & $1(0.6)$ \\
\hline
\end{tabular}

\footnotetext{
\&, loss of clinical or follow-up data. RCC, renal cell carcinoma.
} 
Table S2 Detailed adjunctive therapy and response of grade 4 RCC patients

\begin{tabular}{|c|c|c|}
\hline Systemic treatment & Number of patients & Proportion of treatment subset, \% \\
\hline $\mathrm{CR}$ & 4 & 30.8 \\
\hline PR & 1 & 7.7 \\
\hline SD & 1 & 7.7 \\
\hline \multicolumn{3}{|l|}{ Sorafenib $(n=12)$} \\
\hline $\mathrm{CR}$ & 2 & 16.7 \\
\hline PR & 3 & 25.0 \\
\hline SD & 1 & 8.3 \\
\hline $\mathrm{CR}$ & 1 & 50.0 \\
\hline PD & 1 & 50.0 \\
\hline \multicolumn{3}{|c|}{ Sunitinib + sorafenib $(n=1)$} \\
\hline SD & 1 & 100.0 \\
\hline \multicolumn{3}{|c|}{ Sorafenib + axitinib $(n=1)$} \\
\hline PD & 1 & 100.0 \\
\hline \multicolumn{3}{|l|}{ Interferon $\alpha(\mathrm{n}=2)$} \\
\hline $\mathrm{CR}$ & 1 & 50.0 \\
\hline
\end{tabular}

CR, complete response; PR, partial response; SD, stable disease; PD, progressive disease; RCC, renal cell carcinoma. 